$40(2) \mid 2011$

Varia

\title{
Tourisme communautaire, conflits internes et développement local
}

Turismo comunitario, conflictos internos y desarrollo local

Community tourism, internal conflicts and local development

Julie Carpentier

\section{(2) OpenEdition}

Journals

Edición electrónica

URL: http://journals.openedition.org/bifea/1503

DOI: $10.4000 /$ bifea. 1503

ISSN: 2076-5827

Editor

Institut Français d'Études Andines

Edición impresa

Fecha de publicación: 1 agosto 2011

Paginación: 349-373

ISSN: 0303-7495

Referencia electrónica

Julie Carpentier, «Tourisme communautaire, conflits internes et développement local », Bulletin de I'Institut français d'études andines [En línea], 40 (2) | 2011, Publicado el 01 febrero 2012, consultado el 07 noviembre 2020. URL : http://journals.openedition.org/bifea/1503 ; DOI : https://doi.org/10.4000/ bifea. 1503

\section{(c) $(1)$}

Les contenus du Bulletin de l'Institut français d'études andines sont mis à disposition selon les termes de la licence Creative Commons Attribution - Pas d'Utilisation Commerciale - Pas de Modification 4.0 International. 


\title{
Tourisme communautaire, conflits internes et développement local
}

\author{
Julie Carpentier*
}

\section{Résumé}

Au cours des 15 dernières années, le tourisme communautaire en Amazonie équatorienne a connu un essor sans précédent. Ce fait récent, impliquant une remise en question des pratiques et des savoirs culturels indigènes, amène donc à s'interroger sur les enjeux de cette nouvelle activité. Dans cet article, l'auteur présente les résultats préliminaires d'une étude de terrain réalisée auprès de trois communautés kichwas de la province du Pastaza qui tentent de développer le tourisme dans l'espoir d'améliorer les conditions de vie de leurs habitants. L'article évoque tout d'abord le phénomène touristique en lui-même, ses enjeux et les conflits qu'il peut engendrer au niveau local et interne. L'auteur s'interroge ensuite sur la politique de réappropriation culturelle mise en place dans le cadre du tourisme communautaire. Enfin, le texte aborde les rapports complexes créés par l'entreprise pétrolière Agip, financeur principal des projets touristiques, et s'intéresse aux conséquences de sa présence sur les modes de vie des communautés ainsi que sur sa manière de concevoir un tourisme communautaire local.

Mots clés : Amazonie, Équateur, kichwa, tourisme communautaire, réappropriation culturelle, acculturation, entreprises pétrolières

\section{Turismo comunitario, conflictos internos y desarrollo local}

\section{Resumen}

Durante los últimos 15 años, el turismo comunitario en la Amazonía ecuatoriana se ha desarrollado de manera fulminante. Este nuevo fenómeno modifica las prácticas y sabidurías culturales indígenas y obliga a contemplar los desafíos que esta nueva actividad conlleva. En el presente artículo se proponen

* UMIFRE 17, CNRS-MAEE (IFEA), LESC, Université Paris-Ouest Nanterre La Défense, 200 av. de la République, 92000 Nanterre. E- mail: julie.carpentier@u-paris10.fr 
algunos avances de un estudio de terreno realizado en tres comunidades indígenas kichwas de la provincia del Pastaza que intentan desarrollar el turismo con el sueño de mejorar las condiciones de vida de sus habitantes. En primer lugar, el artículo se interesa por el fenómeno turístico, en los desafíos que plantea, y en los conflictos que puede generar al nivel local e interno. La autora se interesa luego en la política de reapropiación cultural en el marco del turismo comunitario. Finalmente se analizan las relaciones complejas entre las comunidades y la empresa petrolera Agip que financia los proyectos turísticos, y se plantean las consecuencias de su presencia en los modos de vida y la manera de concebir un turismo comunitario local.

Palabras clave: Amazonía, Ecuador, kichwa, turismo comunitario, reapropiación cultural, aculturación, empresas petroleras

\title{
Community tourism, internal conflicts and local development
}

\begin{abstract}
During the last 15 years, community tourism in Ecuadorian Amazonia has experienced a brilliant rise. This recent phenomenon requires a questioning of the practices and native cultural knowledge, and therefore leads to questioning about this challenges of this new activity. In this article, the author presents the preliminary results of a study realized in three kichwa communities of the province of Pastaza who try to develop tourism in order to improve the inhabitants' living conditions. The article is interested first of all in the tourism phenomenon in itself, including the stakes and conflicts which it can generate at a local and internal level. The author then explores the politic of cultural reappropriation generated within the community tourism's framework. Finally, the article focuses on the complex relationship between communities and the oil company Agip, the main financier of these tourism projects. It is interested in the consequences of this presence on the lifestyles and on the way of thinking about local community tourism.
\end{abstract}

Key words: Amazonia, Ecuador, kichwa, community tourism, cultural reappropriation, acculturation, oil companies

\section{INTRODUCTION}

Le tourisme communautaire tient une place particulière en Équateur. Celui-ci a acquis l'image d'un pays pionnier qui développe un tourisme alternatif aux mains des communautés indigènes. En Amérique latine, l'Équateur fut l'un des premiers pays à affirmer une présence indigène à travers la création de fédérations, d'organisations, et à mener un mouvement de revendication qui connut son apogée en 1992, lors de la célèbre marche vers Quito (Sawyer, 1997)1. Cette affirmation identitaire allait de pair avec une volonté politique de participation et de reconnaissance au niveau national et international qui a revendiqué, dès le début des années 1990, le dispositif des «terres indiennes pour la conservation »

1 La Fédération Équatorienne des Indiens (FEI) est fondée en 1944 dans la région andine. Elle avait pour but principal d'implanter un système d'enseignement bilingue et d'améliorer les conditions de vie et de travail des travailleurs des haciendas. Dans la région amazonienne, le processus a commencé dans les années 1960 avec les organisations shuars créées sous l'impulsion des missionnaires salésiens. 
(Dumoulin, 2006). Les politiques indigénistes jouèrent ainsi la carte écologique en concevant leur approche de manière plus environnementaliste. Elles trouvèrent un écho certain dans l'avènement du tourisme durable sur la scène internationale qu'elles surent adapter aux enjeux locaux de développement. La relation conflictuelle qui existait à l'époque entre le tourisme et la conservation se trouvait de facto résolue. Le tourisme communautaire, alliant protection de l'environnement et découverte de la culture, s'est donc très vite retrouvé intégré à la notion d'écotourisme. L'année 2002, proclamée " année internationale de l'écotourisme » par les Nations Unies, fut également, en Équateur, I'année de la reconnaissance du tourisme communautaire comme forme de tourisme à part entière, au même titre que ses homologues du public et du privéz.

Le tourisme, troisième ressource de l'Équateur après le pétrole et la production bananière, se concentre sur un territoire relativement restreint qui possède l'une des plus grandes diversités d'écosystèmes et de cultures au monde. À l'heure où la question environnementale bat son plein, il apparaît comme l'un des enjeux principaux du pays pour les décennies à venir.

Encouragés par un gouvernement désireux de redéfinir sa politique de développement, les projets de tourisme communautaire se sont multipliés ces dernières années. En 2007, la Fédération Plurinationale du Tourisme Communautaire en Équateur (FEPTCE) recensait 11 projets dans la partie amazonienne ; elle en recense 35 en 2010. Cet engouement pourrait nous laisser perplexe mais il faut se rendre à l'évidence : les acteurs qui gravitent autour de ces projets sont nombreux. Qu'ils soient partenaires financiers ou soutiens divers, ces derniers apportent l'argent nécessaire à la construction des sites, aux formations, à l'entretien... en échange de quelques accords implicites censés répondre aux intérêts de chacun. Au cours des 10 dernières années, il faut savoir que les compagnies pétrolières se sont placées sur le marché du tourisme communautaire, répondant ainsi aux besoins de développements locaux tout en s'assurant une présence « tolérée ». Ces « investisseurs » ont un rôle non négligeable dans l'élaboration de ces projets. Ils orientent souvent le discours local tenu par les représentants de la communauté et donc par les habitants. À la fois un discours officiel et officieux qui caractérise le particularisme du tourisme communautaire en Amazonie.

Si le tourisme communautaire en Amazonie équatorienne a suscité l'intérêt scientifique dès les années 1990 (Colvin, 1994 ; Schaller, 1996 ; Wesche, 1993 ; Epler Wood, 1998), il faut néanmoins préciser que peu d'analyses partent de la vision indigène du tourisme (Zeppel, 2007) ou bien mettent en avant le rôle joué par les acteurs extérieurs. Stronza \& Gordillo (2008), dans Community view of ecotourism, se sont intéressés à l'étude d'un échange réalisé entre trois communautés bolivienne, péruvienne et équatorienne en 2003 où l'écotourisme et ses impacts sont analysés du point de vue local. L'ouvrage de Ruiz \& Solis (2007)

2 L'Équateur est ainsi le seul pays au monde qui différencie le tourisme communautaire du privé et du public. Se référer à la « ley de turismo » n²002-97. 
décrit quant à lui le tourisme communautaire en Équateur à travers une analyse à la fois historique du processus et de présentation d'études de cas, révélant le rôle positif des ONG et des agences internationales dans les financements de projet. Rodríguez (1999) analyse pour sa part le succès du lodge Kapawi en Équateur à travers le rôle joué par l'entreprise privée (agence de voyage).

Vue comme un outil de développement alternatif, beaucoup d'études en Équateur ont mis en avant les aspects positifs de cette activité (Drumm, 1991 ; 1998 ; Wesche \& Drumm, 1999 ; Epler Wood, 2002 ; Varga, 2007 ; Ruiz \& Solis, 2007 ; Ruiz et al., 2008). On en retient que le tourisme communautaire ou écotourisme est bénéfique car il permet aux habitants de développer leur propre projet sans dépendre des entreprises d'extraction (Wesche, 1996), il renforce I'organisation communautaire (Coca Pérez, 2007) et favorise l'empowerment (Ruiz \& Hernández, 2010), il permet une autonomie financière plus importante et la création de projets de développement locaux (Drumm, 1991; Wunder, 1996 ; Gorman, 2006 ; Ruiz et al., 2008)... Les auteurs qui ont cherché à décrire les impacts socioculturels négatifs du tourisme sont moins nombreux. Smith (1993), par exemple, a traité de la problématique du développement touristique chez les Huaoranis et, à travers le point de vue des indigènes, des missionnaires, des hôteliers et des « guides », il a pu appréhender la complexité des relations, les risques de conflits et de divisions causés par une telle activité3. Hutchins (2007) montre quant à lui les risques des communautés d'altérer leurs relations avec les communautés voisines ainsi qu'avec leur environnement.

La question du développement (à laquelle est indéniablement lié le tourisme communautaire), et de la présence pétrolière en Équateur et de ses conséquences pour les peuples indigènes a également connu un intérêt grandissant (Kimerling, 1993; Santos Granero, 1996 ; Muratorio, 1996; Narváes, 1996; Rival, 1997 ; Fontaine, 2006). Avalos Mendez (2005), quant à elle, s'est penchée sur la question du tourisme en tant que nouvelle forme de développement, sans toutefois évoquer le rôle des acteurs extérieurs dans cette activité. Pour sa part Trujillo (2001) s'est intéressé aux « agents civilisateurs », acteurs du développement en Amazonie, en accordant une partie minime à la question du tourisme et au folklorisme auquel elle est souvent associée.

L'investissement des entreprises pétrolières dans le développement du tourisme communautaire reste à étudier car il y occupe une place non négligeable : en effet, les clauses des contrats d'exploitation, signés avec les communautés, incluent quasi systématiquement un programme d'éducation et de renforcement identitaire et culturel local, qui se traduit de plus en plus par la construction de projets touristiques locaux.

L'entreprise pétrolière Agip a, de ce point de vue, très bien compris les enjeux liés à l'activité touristique. Présente depuis la fin des années 1980 dans la province du Pastaza, elle a accepté de financer le projet touristique mis en place par

3 Pour un état de la question plus complet, non focalisé sur l'Amazonie équatorienne, voir Stronza (2001; Stronza \& Gordillo, 2008). 
I'Organisation Communautaire Liquino Curaray en 2003. Cette organisation, composée des communautés kichwas d'Atacapi, de Liquino et de San Virgilio considère le tourisme comme un outil de développement local propre, permettant de valoriser la culture de ses habitants tout en assurant à ceux-ci un meilleur avenir. Comment expliquer alors que le site d'Ishkay-Yaku n'ai encore reçu aucun touriste ? Divisions internes, dégradation du site, désintérêt de la population..., ce complexe est pris en contre-exemple dans tout le Pastaza mais ne dissuade pas pour autant les communautés voisines. San Virgilio, malgré cette expérience désastreuse, a choisi de tenter sa chance à nouveau en élaborant un projet, également financé par l'entreprise Agip. Elle reste persuadée que son complexe, lui, marchera.
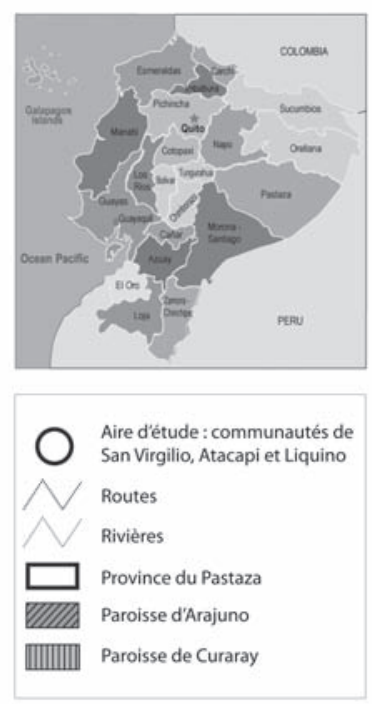

|

Figura 1 - Macro-localisation de la zone d'étude

Source : Ecociencia, élaboré par Chloé Carpentier

L'expérience de ces trois communautés confrontées à de complexes problématiques de développement amène à nous intéresser au rôle joué par la présence pétrolière dans l'élaboration de ces projets de tourisme communautaire ainsi qu'aux enjeux tant économiques qu'identitaires qu'une telle activité implique. Dans cette optique, il convient avant tout de définir deux notions qui reviendront fréquemment tout au long de cet article. Celle de « culture » notamment, que nous entendrons comme culture collective qui forme l'identité d'un peuple et se compose de «l'ensemble des traits distinctifs, spirituels et matériels, intellectuels et affectifs, qui caractérisent une société ou un groupe social » (Unesco). Celle enfin de «tradition » que nous définirons comme un héritage culturel immatériel 
qui se transmet à travers I'histoire, pouvant constituer le vecteur d'une identité à laquelle se rattache un groupe humain.

L'analyse prendra en compte les projets touristiques, en tentant de mesurer les enjeux économiques et politiques qui les sous-tendent ainsi que les raisons qui ont amené la communauté de San Virgilio à construire ses propres « cabañas » et à se détacher du projet initial : Ishkay-Yaku. La question culturelle sera abordée ensuite, à travers le processus de valorisation et de récupération mis en place par l'Organisation Liquino Curaray et les communautés. Enfin, des éléments de reflexion seront livrés sur les conséquences de la présence des entreprises pétrolières (la compagnie Agip dans le cas qui nous intéresse) sur les modes de vie des communautés et sur la manière de concevoir un tourisme local.

\section{LE TOURISME, FER DE LANCE DES COMMUNAUTÉS}

Le tourisme est aujourd'hui perçu comme le fer de lance des communautés. Bien que seuls $6 \%$ des touristes étrangers décident de s'aventurer en Amazonie, bon nombre d'entre elles croient en la réussite de leurs projets, encouragées par le Ministère du Tourisme qui souhaite notamment développer cette activité dans la région4.

Les communautés d'Atacapi, de Liquino et de San Virgilio ont choisi de se lancer dans cette voie en 2003 en créant la «Organisación de las Comunidades Indígenas de la Cabecera del río Curaray Liquino ». Au projet touristique venait s'ajouter celui d'élevage de porcs et de production de citrons destinés à la vente ainsi que celui d'achat de vaches pour chaque famille. Cette volonté de développer un marché local bénéfique à l'ensemble des habitants s'inscrit dans un contexte national et international d'affirmation communautaire et de lutte contre la pauvreté dont se réclame le gouvernement actuel du président Correa.

En 2003, Wilson Santi, président de la communauté de San Virgilio et de l'Organisation, exposa, lors d'une assemblée à Atacapi, le projet communautaire d'un centre touristique : I'accueil de touristes permettrait de récupérer la culture kichwa, de valoriser la production traditionnelle, de renforcer l'unité de chaque communauté, tout en améliorant les conditions de vie de la population locale grâce aux bénéfices générés. Le projet ne reçut tout d'abord pas l'approbation d'Atacapi et de Liquino. Il fallut de longues assemblées afin de convaincre les habitants de se lancer dans l'aventure. Une fois l'accord et les financements obtenus, les travaux commencèrent.

Le projet fut financé partiellement par l'État, les parties prenantes étant le «COnsejo de DEsarollo de las Nacionalidades y Pueblos de Ecuador » (Codenpe),

4 Le Ministère du Tourisme est actuellement en train de développer un plan stratégique de développement du tourisme durable en Équateur appelé « Plandetur 2020 » afin de l'insérer dans la politique d'État et afin qu'il soit un élément dynamisant du développement économique et social. Le «Plandetur 2020 » s'intègre à un plan de développement national plus global appelé «Plan Nacional para el Buen Vivir » qui regroupe tous les ministères. 
I'« Instituto para el ECOdesarollo Regional Amazónico Ecuatoriano » (Ecorae), le Ministère du Tourisme. Mais la compagnie pétrolière Agip a aussi investi à hauteur de 100000 \$ pour la construction du site, lequel comprend 6 « cabañas », un restaurant/cuisine, une maison culturelle, une lagune (artificielle) et un refuge animalier. Afin de gérer cette activité, I'Organisation a employé un coordinateur, d'origine kichwa, qui avait travaillé pour l'Ecorae et I'Organisation des Peuples Indigènes du Pastaza (OPIP).

En 2007, Ishkay-Yaku était inscrit à la FEPTCE, et reconnu par le Ministère du Tourisme. Or, en 2010, les habitants attendent toujours les premiers touristes, bien qu'une fiche du Ministère du Tourisme remplie en 2007 par le président de la FEPTCE indique que le complexe a reçu 60 touristes cette même année (35 nationaux et 25 étrangers venus des EU). Le site est aujourd'hui géré par un gardien, chargé de l'entretien, et de l'aménagement (sans doute pour justifier les financements octroyés5).

L'absence totale de touristes ces dernières années s'explique de plusieurs façons :

- Un investissement limité de la population, la construction du complexe ayant été prise en charge par des entreprises de Puyo, capitale de la province.

- Un rôle contesté de l'Organisation qui parait se satisfaire de cette situation. Le coordinateur du projet touche plus de 2000 \$ par mois, a signé un contrat à durée indéterminée et vient de se faire élire «socio » d'Ishkay-Yaku, responsable en quelque sorte. II est connu pour ses rapports privilégiés avec Agip, son manque de transparence et est totalement opposé à l'intervention de l'État dans les signatures des contrats qui lient compagnies pétrolières et communautés6.

- Une présence importante d'Agip qui finance les écoles, les centres de santé, un médecin, qui paye les membres de l'Organisation et des communautés bien audelà du salaire moyen équatorien, et qui envoie chaque mois de la nourriture (riz, sardines, thon) et de l'alcool... Les besoins des communautés apparaissent donc limités, alors que la majorité de la population voit dans le tourisme une source de revenus non négligeable qu'elle continue d'espérer.

En juillet 2009, la communauté de San Virgilio a fait savoir son désaccord sur le fonctionnement de l'Organisation et son désir de quitter le bureau, suite à I'expérience d'Ishkay-Yaku. La séparation de San Virgilio est clairement due à un conflit ouvert entre le coordinateur et l'actuel président de la communauté, totalement opposés sur la manière de fonctionner tant sur le plan de l'Organisation que sur celui du projet touristique en lui même. Le coordinateur, accusé de

5 En janvier 2010, chaque « cabaña » a été équipée d'un mini-frigo et en mai, les membres du bureau ont tous reçu un talkie-walkie.

6 À la suite de nombreuses plaintes déposées par les communautés indigènes, l'État tente actuellement de s'immiscer davantage dans les contrats signés avec les compagnies pétrolières. De nombreuses réunions ont été organisées ces derniers mois entre les différents protagonistes, sans grand résultat, si ce n'est de multiplier les conflits entre les communautés elles mêmes. En janvier 2010, I'État a demandé aux compagnies de leur envoyer les fameux contrats. Au mois d'avril, ils n'avaient toujours rien reçu. 
malversations et de mauvaise gestion ne put cependant être licencié, et pis, il tient actuellement une place centrale au sein de l'Organisation. San Virgilio s'est donc retirée administrativement (la compagnie Agip refusant son départ), mais garde toutefois un droit de regard sur celle-ci et devrait normalement obtenir la présidence en décembre 2010...7 En attendant, elle possède aujourd'hui son propre bureau à Puyo et s'investit depuis peu dans un nouveau projet touristique, cette fois-ci propre à sa communauté.

Dans ce projet, les travaux sont effectués par les habitants eux-mêmes, financés à hauteur de 60000 \$. La mairie d'Arajuno (chef lieu de canton) a investi 15000 \$ à travers le projet du «Corredor Central»8. Les trois-quarts restants sont, quant à eux, financés par... la compagnie Agip. Au cours de la troisième semaine de mai 2010, la communauté renouvelait son contrat avec l'entreprise pétrolière, espérant obtenir davantage de financements pour poursuivre la construction du site.

Ce dernier n'est accessible que par un chemin de $10 \mathrm{~km}$ très vallonné et propose des activités (cascade, saladero, pierre sacrée, lagune...) qui nécessitent plusieurs heures de marche. Cela suppose un public plutôt jeune, en bonne santé physique, tout comme la majorité des sites de tourisme communautaire qui n'ont aucune facilité d'accès, la voie fluviale étant peu utilisable dans la province du Pastaza.

La communauté espère accueillir ses premiers touristes dans les prochains mois, aidée depuis peu par un ingénieur en tourisme équatorien chargé de développer le projet, notamment la partie promotionnelle qui fait le plus souvent défaut. Mais elle accueille déjà différents étudiants en tourisme chargés d'inventorier les activités envisageables pour confectionner des « packs » touristiques (cascade, saladero, pierre sacrée, lagune...), relever les points GPS pour créer de nouveaux sentiers accessibles et élaborer une nouvelle politique de traitement des déchets. Autant de changements qui viennent profondément modifier l'organisation même de la communauté qui possède peu d'expérience dans le domaine touristique et s'appuie sur des acteurs extérieurs pour développer son nouveau projet qu'elle souhaite voir se concrétiser.

L'échec d'Ishkay-Yaku n'est en effet pas un cas isolé et révèle les difficultés rencontrées par les communautés pour développer leurs projets : un manque de formation et d'organisation certain auquel s'ajoutent des conflits internes provoqués par une arrivée massive d'argent et des intérêts personnels divergents. La « séparation » de San Virgilio et le développement d'un nouveau projet touristique montre l'importance donnée à cette nouvelle activité à travers laquelle la population fonde une grande partie de ses espoirs. L'inquiétude repose à présent

7 Les élections ont eu lieu en mars 2011 et la communauté de San Virgilio n'a pas été conviée à I'assemblée. La communauté d'Atacapi a de nouveau obtenu la présidence.

8 ONG qui propose des soutiens financiers sur une petite portion du pays qui relie Portoviejo (côte) à Arajuno (Pastaza) afin d'aider les communautés à développer des projets soutenables liés à l'agriculture, aux ressources naturelles et à la diversité locale. L'exécution des projets est à la charge du Ministère de l'Agriculture, de l'Élevage, de l'Aquaculture et de la Pêche qui délègue aux bureaux régionaux du Corredor Central situé à Portoviejo, Latacunga et Puyo. Le financement provient du Fond International du Développement Agricole (FIDA). Ce projet regroupe actuellement 6 communautés dans le canton d'Arajuno qui développent le tourisme, chacune ayant touché 15000 euros. 
essentiellement sur les impacts culturels négatifs que le tourisme peut engendrer et sur les incertitudes quant à la réussite du projet. Afin de gérer au mieux ce genre de difficultés, Atacapi, Liquino et San Virgilio se trouvent dans un processus de socialisation qui intègre avant tout récupération et revalorisation culturelles, à travers des formations et l'enseignement des anciens de la communauté.

\section{LE TOURISME COMMUNAUTAIRE, UN OUTIL DE RÉAPPROPRIATION CULTURELLE ?}

Depuis plusieurs générations, la culture kichwa connaît une crise identitaire importante liée à l'intensification des contacts avec le monde extérieur. L'histoire de l'Amazonie équatorienne est rythmée par un processus d'acculturation, de transformations sociales et culturelles et de folklorisation des peuples indigènes qui remontent au XVI e siècle. La colonisation, l'évangélisation et l'arrivée des entreprises d'extractions ont fortement modifié les modes de vie (Muratorio, 1991 ; 1994 ; Santos Granero, 1992 ; 1994 ; 1996). Depuis les années 1970, l'influence du monde urbain, bien visible, se traduit par l'achat de matières premières ou de produits manufacturés auquel s'ajoute le départ des jeunes vers les villes. Mais c'est au niveau culturel que les changements les plus importants peuvent s'observer. Les pratiques traditionnelles ont parfois disparu au profit d'une culture intégrée, alliant savoirs ancestraux et apports du monde occidental. Actuellement, la création de l'Organisation communautaire, la construction des sites d'Ishkay-Yaku et de San Virgilio ont réactivé certaines pratiques, notamment culturelles. Dans quel but? Et sous quelle forme?

La mise en place du tourisme communautaire est un processus complexe car elle implique la construction d'une authenticité culturelle permettant de rejeter l'idée de «folklorisation » systématiquement attachée à cette activité. Selon Del Campo Tejedor (2009), I'authenticité, dans ce cas précis, requiert quatre éléments : le traditionnel, l'exotisme, la pureté et la sincérité. En cherchant à répondre à un public donné, les communautés choisissent ce qu'elles veulent donner à voir et basent leur politique de récupération culturelle à la fois sur l'aspect matériel (objets, ornements, construction...), mais également sur l'aspect immatériel (chamanisme, chants, langue). Ces marqueurs identitaires répondent donc à une demande touristique (les incorporant indéniablement à un processus commercial), tout en participant à une redéfinition du patrimoine culturel local avec pour objectifs la cohésion et le renforcement des liens sociaux.

La réappropriation des savoirs culturels peut s'effectuer sous diverses formes qui s'articulent autour de deux axes principaux : les formations éducatives d'une part et la transmission par les doyens d'autre part. Son succès dépend avant tout de l'ensemble de connaissances ou d'aptitudes reproductibles déjà acquises et héritées par certains membres de la communauté et de la capacité à intégrer la jeune génération dans ce processus. 


\section{1. Les formations : un apprentissage des savoirs culturels}

La communauté d'Atacapi fut créée en 2001 et se compose d'une population jeune (30-40 ans) qui possède un savoir culturel limité. Afin de pouvoir investir les habitants au projet touristique, et plus précisément les femmes, I'Organisation a choisi de financer deux formations consacrées à la fabrication de céramiques, de colliers, bracelets et boucles d'oreilles9.

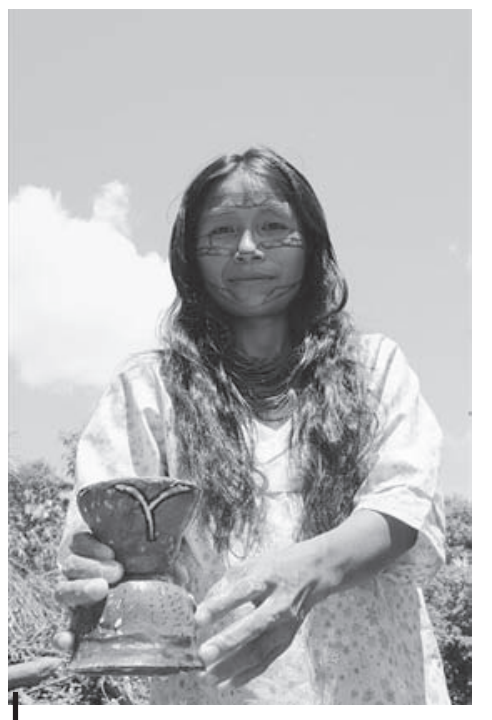

Figure 2 - Femme Kichwa servant la chicha

Photo : Julie Carpentier

Le premier cours fut dispensé par une habitante locale et s'est déroulé sur une semaine. Les femmes y ont essentiellement appris à diversifier, voir à complexifier les motifs peints et à améliorer leurs techniques de fabrication de céramique. Toutes les céramiques, une fois réalisées, ont été présentées à la communauté. Le projet a été applaudi par le président qui a souligné l'active participation tout en rappelant l'importance de valoriser les savoirs traditionnels kichwas et... de nouveau, la chicha est servie dans des « mocahuas $» 10$. Cette socialisation effectuée auprès de la population joue un rôle important au niveau de la prise de conscience collective et de l'investissement de chacun au projet.

Le deuxième cours a eut lieu dès le lendemain. C'est une employée de la municipalité de Puyo, originaire de Sarayaku (Kichwa), qui est venue enseigner lestechniques d'assemblages de perles pour la création de colliers, de bracelets et de boucles d'oreilles. À la différence des céramiques, les femmes d'Atacapi n'avaient aucune connaissance dans ce domaine et ont appris en une semaine à réaliser de nombreuses sortes de bijoux. Du fil, une aiguille et des perles en plastique importées de Puyo, auraient contribué à la récupération de pratiques traditionnelles kichwas. Bien qu'il fasse aujourd'hui partie de l'ornementation corporelle, un collier fait avec des perles en plastique, demande beaucoup moins de temps de réalisation qu'un collier traditionnel réalisé à l'aide de graines récoltées dans la forêt, percées à la main et assemblées sur un fil en fibre naturelle. Tout dépend de la définition que l'on souhaite donner au mot «tradition», chaque culture évoluant et s'adaptant au cours du temps, I'influence des contacts extérieurs et de l'assimilation culturelle jouant un rôle essentiel dans ce processus. Selon G. Lenclud (1987) « la traditionnalité est une condition de changement ». Ainsi, plus une société a les moyens de reproduire le passé, plus elle est apte à perpétrer le changement. Elle justifie alors, d'une certaine manière, son état contemporain en affirmant sa différence à travers, par exemple, l'élaboration d'un projet de tourisme communautaire. La question reste

9 Ces formations ont également été proposées dans la communauté de Liquino deux semaines plus tard.

10 La chicha est la boisson locale faite à base de yuca fermenté. Elle est traditionnellement servie par les femmes dans des bols en terre cuite ornés de dessins. 
de savoir à partir de quels éléments mémoriels la réappropriation culturelle trouve son origine, et quelle authenticité faut-il lui accorder lorsqu'elle s'inscrit dans un programme de développement touristique à visée économique?

\section{2. Le rôle des doyens dans la transmission des savoirs}

Les doyens des communautés sont incontournables. Leurs connaissances sont beaucoup plus importantes que celles des jeunes, trés acculturés du fait d'une accélération sans précédent de leurs relations avec le monde extérieur. La prise de conscience identitaire de la génération des 30-40 ans, qui est à l'origine de ces projets de tourisme communautaire, est souvent le fait de personnes qui ont été séparées de leur communauté d'origine, ont voyagé et souvent étudié. C'est par exemple le cas des enfants de Bolívar, doyen de la communauté de San Virgilio, élevés en ville, à Puyo, et dont la rupture culturelle s'est traduite par une perte de repères et un désir de retour aux sources.

À San Virgilio, la présence de Bolívar (68 ans) et celle de sa femme, jouent un rôle très important en matière de transmission culturelle. Elle s'effectue de manière orale, en diverses occasions, essentiellement lors des assemblées qui restent un moment privilégié de partage et d'échanges11. Bolívar n'est autre que le dirigeant en charge du tourisme au sein de la communauté de San Virgilio et cette fonction révèle le rôle central qu'il joue au sein de ce processus de transmission culturelle. L'originalité réside également dans le mélange de deux cultures: shuar et kichwa, de part l'origine métisse de Bolívar, bien visible dans tous les aspects de la vie communautaire. Les deux maisons communautaires sont construites sur le modèle shuar, chaque morceau de chonta composant les murs étant taillé en forme de flèche par Bolívar lui-même. Sa femme, Kichwa, réalise des colliers shuars, reconnaissables par leurs multiples couleurs. Quant à Bolívar, il continue à chanter en shuar, transmettant le goût de la musique et enseignant la flûte.

«Mi padre tiene una historia muy larga, tantas cosas que nos ha conversado, muchas, muchas, muchas, como andaban a la casería, cuál tío casi le comió el tigre, como vinieron por acá, todo eso... » (Wilson Santi).

Le tourisme communautaire est très généralement associé à un tourisme culturel où l'idée d'inter-culturalité s'impose. La nécessité de connaître son histoire, sa langue et sa culture fait également partie de ce processus, généralement beaucoup mieux maîtrisé par les anciens.

11 Traditionnellement, les femmes se lèvent à $3 \mathrm{~h}$ pour préparer la guayusa, thé à base de feuilles de guayusa connues pour leur forte teneur en caféine. Toute la famille se réunit autour du feu et discute de choses et d'autres (rêves, organisation de la journée, histoires, légendes...) jusqu'au lever du jour. Ces moments sont l'occasion de transmettre la culture et la tradition aux plus jeunes, qui eux-mêmes les transmettront à leurs enfants. 


\section{3. La jeune génération : un élément clé pour l'avenir}

La question des jeunes apparait en effet centrale dans ce nouveau mouvement culturel. Les parents s'inquiètent de l'éloignement de leurs enfants, que ce soit au niveau culturel ou physique : « los niños, especialmente, se están alejándose » (Wilfrido Santi). S'intéressant davantage au dernier téléphone portable en vente qu'aux légendes de ses aînés, la majorité des jeunes profite de chaque occasion possible pour partir à Puyo, y trouver un emploi, et s'y installer. Le tourisme est donc apparu comme une solution afin que ces derniers n'abandonnent pas leur pays et s'intéressent tant à leur culture qu'aux nouvelles technologies, tout cela dans la perspective d'emplois autres que ceux qui leur sont proposés en ville.

La question de la langue reste l'un des points les plus sensibles. Ainsi, la langue espagnole imposée à l'école a eu pour conséquence fâcheuse une perte d'identité profonde chez les jeunes, sachant que la majorité des parents la tansmet aux enfants dès leur naissance afin de limiter leurs difficultés scolaires. De ce fait, aujourd'hui, la jeunesse ne parle plus kichwa ou du moins très mal et refuse de s'exprimer autrement qu'en castillan. Le projet touristique a donc le mérite de faire prendre conscience aux parents de ce problème, la question de la valorisation culturelle étant intimement liée à l'usage de sa propre langue.

«Nuestros hijos ya no piensan en la cultura. Como están estudiando, quieren cambiarse. Pero yo siempre he dicho a mis hijos yo he cambiado pero siempre hablo en kichwa. Mi hijo casi no escuchaba kichwa, no hablaba, solo podíamos hablar castellano. Mis sobrinos también, escuchan todo pero no pueden hablar » (Isaac Santi).

Un point concerne également l'image que se font les jeunes de leur culture et de leur origine. II existe un ostracisme très fort envers les populations indigènes qui explique en partie le rejet de certaines pratiques. Dans un contexte comme

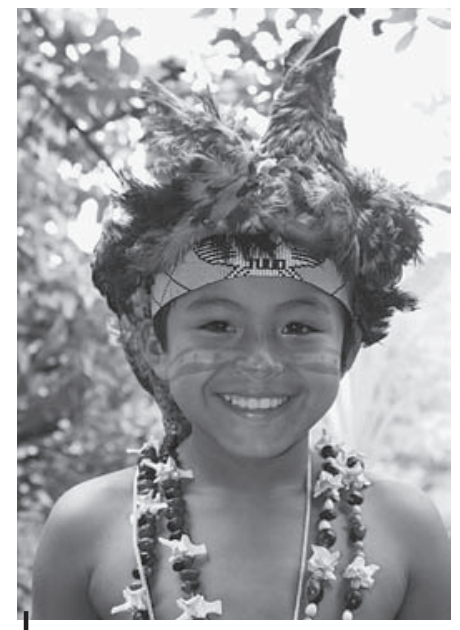

Figure 3 - Jeune danseur Kichwa

Photo : Julie Carpentier celui-ci, il apparaît d'autant plus difficile de mettre en place une politique de récupération et de valorisation culturelle. Comme beaucoup d'adolescents rejettent leur origine kichwa quand ils arrivent en ville, le tourisme est vu par les dirigeants comme un moyen de récupérer ce sentiment de fierté en stimulant les jeunes et en leur offrant l'opportunité de participer directement au projet, espérant ainsi limiter l'émigration vers les villes.

" Últimamente estamos para ver lo que es nuestra costumbre. Porque nuestros jóvenes se han olvidado, inclusive yo me estoy olvidando.(...). Vamos a ver si de aquí, unos pocos tiempos (...) con las cabañas turísticas que estamos haciendo, yo creo que allí tenemos un futuro » (Wilson Santi).

Aussi, le groupe de danse de San Virgilio fait partie de ces initiatives mises en place pour faire participer les jeunes. La partie musicale se voulant à la fois traditionnelle et contemporaine permet de valoriser leurs goûts. Certains écrivent même leurs textes qui parlent de la vie locale et du 
projet touristique, exprimant ainsi leurs sentiments et leur volonté de s'investir dans la communauté.

« estoy felicitando últimamente hace unos 6 meses que empezamos con los niños. Ya veo bien emocionados » (Wilson Santi).

Le projet touristique apparaît donc comme un outil qui permet de travailler sur d'autres projets, lesquels visent à améliorer le « bien être » de la communauté, notamment en valorisant une culture en perdition et en s'affirmant en tant qu'indigène sur la scène internationale. Avec de nouvelles opportunités : rassembler les habitants autour d'un projet et d'une culture commune, assurer des emplois locaux et des revenus supplémentaires.

\section{LA PRÉSENCE DE LA COMPAGNIE PÉTROLIÈRE AGIP ET SES CONSÉQUENCES SUR LE «BIEN ÊTRE » DE LA COMMUNAUTÉ : COMMENT CONCEVOIR LE TOURISME ?}

La notion de «Sumak Kawsay » chez les communautés indigènes, que I'on pourrait traduire par «bonheur/bien-être », représente en quelque sorte l'indice de développement local et est constamment employée par les indigènes comme référent principal au même titre que le PIB dans les différents pays du monde (Ramírez, 2009a)12. Reconnu dans la constitution équatorienne, le concept de «Sumak Kawsay » se définit en quelque sorte par le point d'union entre les droits de l'homme et les droits de la nature. Les indigènes n'emploient que très rarement le terme de « développement » qu'ils définissent comme «la vision occidentale du progrès » se traduisant, selon leur conception, par l'exploitation massive de leurs terres et le non respect de la Pachamama, la Terre-Mère.

\section{1. Historique}

La création de la «Organisación de las Comunidades Indígenas de la Cabecera del río Curaray Liquino », peut difficilement se comprendre sans une prise en compte du contexte politique et économique de l'époque. Dans le processus de distribution de terres octroyées par l'État à des entreprises pétrolières étrangères dès les années 1970, le bloc 10 (200 000 ha), situé dans la province du Pastaza, fut attribué à l'entreprise ARCO Oriente-Agip Oil Ecuador en 1988, les modalités du contrat liées aux prestations de service (explorations-exploitations des hydrocarbures) ayant été signées avec l'entreprise étatique Petroecuador.

Jusqu'en février 2000, ARCO Oriente assumait les opérations du bloc. Elle a ensuite vendu ses parts à Agip Oil Ecuador, filiale du groupe italien ENI, I'actuel

12 Le gouvernement équatorien s'est également approprié cette notion pour appuyer sa nouvelle ligne politique à travers le « Plan Nacional para el Buen Vivir, 2009-2013 » valorisant la plurinationalité et l'interculturalité. 

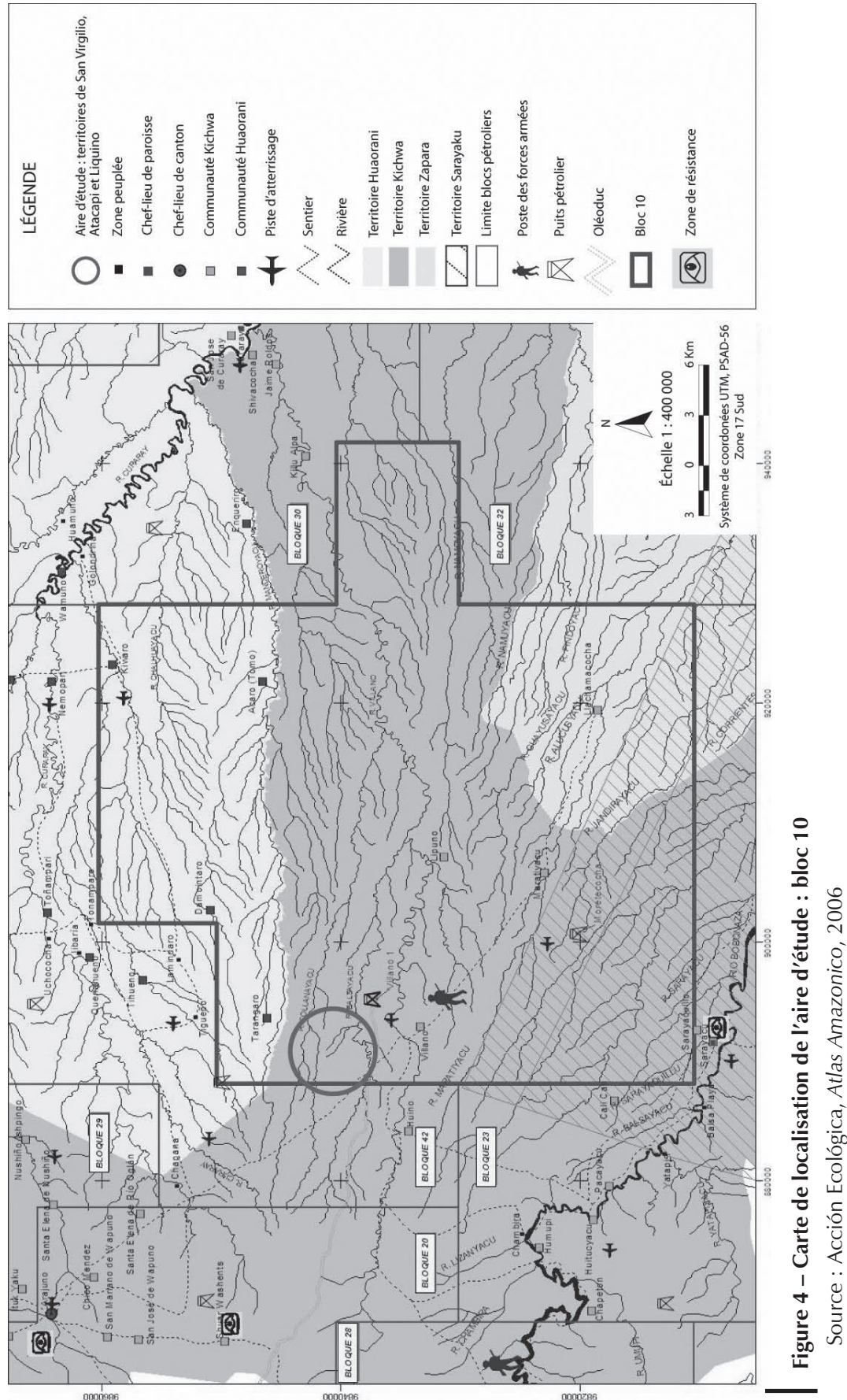
opérateur. Au mois de mars 2000, la communauté kichwa de San Virgilio s'opposait à l'entreprise. Cet affrontement s'inscrit dans une double dimension qui s'articule, d'un côté, autour d'une campagne internationale, appuyée par la Confédération des Nationalités Indigènes de I'Amazonie Équatorienne (Confeniae) consistant à dénoncer les activités de l'entreprise, et, d'un autre côté, autour d'une demande de protection émise devant la cour de justice du Pastaza, rejetée en première instance (Fontaine, 2004).

Jusqu'à aujourd'hui, les motifs de cet affrontement ne sont pas clairs. Cependant, on peut entrevoir une réelle volonté, de la part des protagonistes, d'être reconnus comme interlocuteurs valables, autant que I'Asodira (Asociación de Indígenas Independientes de la Región Amazónica), qui bénéficiait de nombreux avantages auprès d'Agip. Cette association, créée en 1993, aurait elle-même été « mise en place », de manière officieuse, par les pétroliers. Cela permettait à l'entreprise de rester présente au sein de I'OPIP, avec qui elle avait rompu le dialogue depuis 1992, tout en renforçant sa politique de division au sein des communautés, dans le but de mieux contrôler la région (Acción Ecológica, 2006). En février 2008, le président de I'ASODIRA écrivait:

«Denunciamos a la empresa Agip, por su constante labor divisionista, acción que sistemáticamente logra su nefasto objetivo dividiendo la Organización Comunitaria; así el 22 de mayo de 1998 se forma Wichucachi, el 22 de enero del 2003 se forma Riveras Curaray Liquino; y últimamente con la manipulación de AGIP de forma ilegal se conforma el Pueblo Ancestral Huito con las comunidades Púal, Amarún Cocha, Elena y Huito, con la utilización de argucias divisionistas para debilitar ASODIRA y devastar la riqueza natural de nuestros territorios13».

En 1988, la communauté de San Virgilio a signé un contrat de 30 ans en échange de quelques projets liés à l'éducation (matériel, uniformes, mobilier...) et à la santé (poste médical)14. La non-intervention du gouvernement dans ces deux domaines a permis aux compagnies de s'imposer comme les nouveaux mécènes 15 . Leur venue est généralement plutôt appréciée car significative d'amélioration des conditions de vie, d'une meilleure éducation et d'un service médical très souvent inexistant.

«Cuando vino la compañía, ella asumió y hizo la ASODIRA, ellos dijeron "venga, aquí trabajamos todos", pero yo no afilié, yo prefería trabajar independiente. La compañía vino aquí para pedir autorización, pero yo no

13 Eusebio Mayancha Andi. A la colectividad del mundo en busca de conservar la vida de nuestros territorios, Puyo, 21 de febrero del 2008. http://asambleaconstituyente.gov.ec/blogs/francisco cisneros/2008/02/28/foros-ciudadanos-en-orellana-y-sucumbios/

14 La compagnie Agip bénéficie d'un contrat de prestation de service, lequel I'engage à participer au développement des communautés du bloc 10 (éducation, santé, projets productifs...). Le coût financier est néanmoins entièrement pris en charge par l'État.

15 Le gouvernement du président Correa met néanmoins l'accent sur l'éducation et la santé, la situation tendant à s'améliorer dans les communautés amazoniennes où l'État tente de reprendre la main sur une région totalement abandonnée par les précédents gouvernements. 
le daba, nada. Les dije que tienen que irse, que nos van a molestar (...) eso duró meses y meses. Por último hicimos unos papeles, todo, allí decidí trabajar con la compañía. Decían que nos van a ayudar. Hicieron la posta médica, baños y ayudaron con los materiales para la escuela(...) Cuando se iban de allí se acababa lo que ellos tenían que ayudar a nosotros. "Ya" dije, "tráigame este papel que lo firmo para que ustedes pueden mejorar este pueblo" » (Bolívar Santi).

Les habitants, pourtant conscients des dégâts que les pétroliers peuvent occasionner, ne se rendent pas toujours compte des conséquences que leur présence peut engendrer au niveau culturel, social et sanitaire, notamment à long terme. L'exploitation pétrolière dans la province du Pastaza est en augmentation constante, expliquant ainsi les pressions exercées sur les communautés refusant leur établissement et cette nouvelle politique de division mise en place pour mieux contrôler les communautés16. La création de l'Organisation Liquino Curaray est un exemple parfait des conséquences de la présence de la compagnie Agip sur ce territoire. En proposant aux communautés d'Atacapi et Liquino de s'allier, San Virgilio souhaitait obtenir des contreparties intéressantes qui lui auraient permis d'améliorer ses conditions de vie. Le tourisme est apparu comme la solution à tous les problèmes. Au final, le projet n'a jamais fonctionné, les conflits internes à l'Organisation n'ont fait que s'accentuer et Agip est parvenue à manipuler la population, laquelle, aveuglée par la multiplication des projets de développement, I'argent, la nourriture et l'alcool distribués, semble se satisfaire de cette situation, laquelle est néanmoins très compliquée. La question reste en effet de savoir quelles sont les alternatives qui s'offrent aux communautés et de quels moyens elles disposent réellement pour lutter contre ces entreprises qui ont un budget souvent supérieur à ceux des États les plus riches du monde. Les pétroliers prennent en charge (quand ils respectent les contrats) des besoins primaires (éducation, santé) que le gouvernement devrait couvrir. On se retrouve ainsi dans une situation inverse où le «bien être » de la communauté est dû, en partie, à la présence de ces compagnies qui viennent combler l'absence de l'État et proposer des emplois rémunérés, la main-d'œuvre pétrolière se révélant majoritairement indigène.

\section{2. De bienfaiteur à perturbateur, la compagnie Agip se fait entendre}

La présence de la compagnie Agip, de par les impacts sociaux, culturels et environnementaux qu'elle engendre, apparaît pourtant de plus en plus contestée. Ainsi, chaque jour, la communauté de San Virgilio est victime de la présence de l'entreprise pétrolière : 10 minutes, c'est exactement le temps qui sépare le passage de chaque hélicoptère qui survole la communauté 7 jours sur 7 , et ce, de

16 L'entreprise Agip et les habitants de Sarayaku (Pastaza) ont connu de nombreux affrontements au cours de l'année 2010, ces derniers refusant catégoriquement l'exploitation de leurs terres. 
9h à $17 \mathrm{~h}$, afin de transporter de gros conteneurs depuis le Centre de Facilité de Production (CPF) jusqu'au puits de Villano.

«Me disturbe el helicóptero que pasa. Mucha bulla (...) ya no deja conversar. Yo con los de la Agip, siempre discuto: “¿Por qué no pasan por otro lado? Solo por aquí" » (Bolívar Santi).

Le thème de la présence des hélicoptères fut certainement le plus traité au cours de notre séjour, ce qui révèle le grave impact que leurs passages engendrent sur le plan sonore. Ils provoquent également des émissions de gaz dans les rivières, dans la forêt, sur les cultures et les répercussions négatives se font sentir : les animaux ont fui, les poissons sont moins nombreux et le yuca ne pousse plus comme avant. La communauté a demandé qu'une étude de l'eau de la rivière soit effectuée. Les premières analyses devaient être réalisées dans une Université de Quito ; les résultats ne sont jamais arrivés. Les deuxièmes échantillons ont été prélevés par la compagnie elle-même qui a assuré la communauté de la non contamination de leur rivière : les habitants doutent de la véracité de ces propos.

Il ne s'agit malheureusement pas des seuls éléments perturbateurs : les impacts culturels, moins visibles, provoquent également des dégâts importants.

Yana Rumi est la pierre sacrée de la communauté de San Virgilio. Depuis plusieurs générations, et ce bien avant l'arrivée du père de Bolívar17, cette pierre était un lieu sacré où les habitants, et plus spécifiquement les chamans, réalisaient leurs rituels.

« Nosotros teníamos una piedra sagrada, una piedra inmensa, donde que nuestros antiguos hacían su danza e iban a soñar tomando huanto, ayahuasca » (Wilfrido Santi).

Aujourd'hui l'oléoduc passe à moins de 10 mètres de la pierre. Bolívar, qui paradoxalement a travaillé à sa construction, s'indigne aujourd'hui des conséquences que son passage a provoquées sur Yana Rumi, n'ayant obtenu, pour seul lot de consolation, qu'un passage sous l'oléoduc pour accéder à la pierre.

«Yo trabajé dos meses por la compañía para construir el oleoducto. Yo tenía

42 años. Me dijimos que a este edad no cogen para este trabajo » (Bolívar).

Si au cours des 20 dernières années, la présence de l'oléoduc ne semblait pas tant perturber les habitants de San Virgilio, le processus de revendication culturelle et le projet touristique sont venus modifier la donne. La présence de l'oléoduc est aujourd'hui ressentie comme une attaque directe à la population, Yana Rumi représentant le symbole de la culture locale.

« Nos destruyeron nuestra cultura donde que nuestros ancestros, nuestros mayores lo hacían su tradición en este sector (...) Ahora es una piedra muerta »(Wilfrido Santi).

${ }^{17}$ Le territoire de San Virgilio appartenait autrefois aux Zapara qui furent repoussés au début du $20^{\mathrm{e}}$ siècle après l'arrivée des premiers colons et des missionnaires. De nombreuses céramiques ont été retrouvées et une légende, racontée par une vieille femme zapara ayant vécu avec la famille de Bolívar, explique qu'une grosse cloche en or serait enterrée non loin. 
L'histoire de Yana Rumi fait partie intégrante du patrimoine local. Bolívar aime à raconter ses rencontres avec les esprits de la forêt : comment Sacha Warmi a tenté de le tromper pour pouvoir l'épouser, comment il s'est transformé en boa pour vaincre une maladie ou encore de quelle manière Sacha Runa a tenté de tuer son beau-frère alors qu'ils revenaient tous deux de la chasse 18 .

« Nos hacía soñar que ella era una mujer, es una mujer esa Yana Rumi. Yo siempre me iba allá. Cuando llegó la empresa, pasó allí. Nosotros pelamos por la piedra y ahora es así como cimento. No se moja como era antes. Hasta el color se iba cambiando. Entonces mi padre denunció a Italia, con ayuda enviemos un escrito a Estados Unidos... pero empresa es empresa, para que decir? » (Wilson Santi).

La pierre sacrée n'est pas uniquement un enjeu culturel pour la communauté. Qu'elle se meure et ne permette plus de rêver et de partager avec l'esprit Yana Rumi est une catastrophe certaine pour la population. Elle réalise aujourd'hui les conséquences du contrat signé avec I'entreprise qui lui a donné I'autorisation d'entrer sur leur territoire. Au-delà de l'aspect culturel, il s'agit également d'un enjeu territorial important, la pierre sacrée étant le symbole de la communauté. II y a quelques mois, la compagnie a directement profané la pierre en gravant son nom dessus, imposant ainsi sa marque de propriété.

« Habían escrito: COMPAÑÍA AGIP en la piedra » (Bolívar Santi).

Cette provocation va bien au-delà d'un acte purement gratuit mais consiste à rappeler de manière concrète qui est le propriétaire des lieux. Profaner cette pierre sacrée n'a fait que raviver les rancœurs des habitants qui depuis s'organisent afin de la protéger. Chaque semaine, un membre de la communauté s'y rend, déblaie les alentours, vérifie son état et passe au camp de Villano, armé de sa carabine pour, à son tour, affirmer sa présence. Les conflits s'apaisent à coup de financements de projets mais l'agacement grandit dans la communauté qui prend de plus en plus conscience des conséquences de la présence d'Agip sur ses terres. Malgré les impacts provoqués par sa présence, la compagnie Agip souhaite multiplier ses puits d'exploitations.

« Ahora escuchamos que van a perforar el pozo cerca que pasa por la comunidad. Eso nos va a afectar más grande. Dicen que no van a contaminar por acá pero solamente el río que se va por allá a Ishkay-Yaku » (Isaac Santi).

La rivière qui traverse le centre « écotouristique » d'Ishkay-Yaku devrait prochainement être contaminée par la présence du nouveau puits d'extraction d'Agip, la compagnie n'attendant que l'autorisation du coordinateur de I'Organisation Liquino-Curaray pour commencer l'exploitation. II est cependant difficile d'imaginer que la communauté de San Virgilio ne soit pas directement concernée, la nappe de pétrole se situant au centre du lieu de vie de la communauté. Malgré les conséquences désastreuses que cela pourrait engendrer,

18 Sacha Warmi et Sacha Runa sont les esprits de la forêt, homme (Runa) et femme (Warmi). 
le fils de Bolívar, les bottes des pétroliers au pied, contredit son père qui se bat contre la présence d'Agip, en expliquant :

« apoyan a la educación, en algunos proyectos, los uniformes que nos dan, y nunca hemos discutido con la empresa como Villano, compa de la Asodira. Siempre hemos trabajado coordinando ».

Que la communauté soit directement victime de la présence d'Agip est une chose certaine. Qu'elle se soit toujours battue contre sa présence en est une autre. Le projet touristique apparaît comme une alternative de développement que la communauté souhaite voir fonctionner. Mais alors : dans quelles conditions ? II ne faut pas oublier que le projet a été financé au trois quarts par la compagnie. Bien que les dirigeants de la communauté de San Virgilio se plaignent sans cesse de la présence d'Agip, leurs rapports et leurs relations avec l'entreprise pétrolière restent très ambigus. II est de ce point de vue difficile d'envisager que le tourisme communautaire, dans ce cas concret, puisse servir de processus d'indépendance. L'adaptation du discours indigène auprès des différentes instances privées et publiques au niveau national et international (État, missionnaires, ONG, compagnies pétrolières ou minières...) conforte l'idée d'un certain pragmatisme qui consiste à mettre en avant sa « différence » et espérer ainsi un accès corporatiste aux ressources institutionnelles et économiques, lié généralement aux compagnies pétrolières dans le cas amazonien (Ramírez, 2009b). Les communautés ont besoin de financements extérieurs afin de développer leurs projets. Ceci permet aux entreprises pétrolières de s'implanter en imposant l'idée que les communautés ne peuvent rien faire sans leur soutien, signifiant par là que le « développement » local passe obligatoirement par un accord préalable avec la compagnie pétrolière. Mais l'inquiétude majeure pour les communautés n'est absolument pas liée à I'investissement d'Agip dans ces projets de tourisme communautaire, bien au contraire, mais plutôt aux impacts que sa présence entraîne : comment est-il possible d'envisager un tourisme communautaire dans de telles circonstances?

\section{3. De l'écotourisme au « toxi-tourisme", ou comment modifier I'imaginaire touristique amazonien}

La présence des pétroliers paraît souvent incompatible avec la vision idéalisée de I'Amazonie que les populations elles-mêmes continuent de perpétuer, à savoir cette image de la forêt où la nature reprend ses droits, où les bruits sont ceux des rivières et des cascades, des insectes, des animaux, du vent et de la pluie, où les peuples autochtones vivent en harmonie avec la nature : une réalité parmi d'autres, celle majoritairement véhiculée par les agences touristiques. La face cachée, celle d'un territoire exploité d'où les animaux ont fui, d'où les insectes ont disparu, où les maladies cancéreuses se sont multipliées et où les problèmes sociaux sont décuplés, est rarement mise en avant, et pour cause : le tourisme donne du rêve, plonge dans un autre univers, fait découvrir des paysages, des cultures qui permettent de s'évader du quotidien et qui surtout, procure un bien-être. 
Aussi, existe t-il, depuis les cinq dernières années, un intérêt grandissant pour les Toxic-Tours initiés par des organismes ou associations majoritairement écologistes cherchant à éveiller les consciences aux problèmes environnementaux, notamment dans les régions de Sucumbios et d'Orellana19. Si les années 1990 ont été rythmées par les notions de développement durable, d'écotourisme, d'ethnotourisme... à travers lesquelles l'environnement était valorisé en tant que «zone » à protéger, la nouvelle tendance diffère dans son approche. Elle ne cherche pas à montrer le « paradis » amazonien à protéger, mais suit les routes des pétroliers, montrant la contamination provoquée par les exploitations et une autre réalité de la vie des peuples autochtones. Un tourisme « choc » en quelque sorte. De l'éco-tourisme vendeur de rêve, nous passons au toxi-tourisme vendeur d'une réalité tragique, modifiant ainsi tout l'imaginaire lié à la forêt amazonienne, un des poumons de I'humanité. L'écotourisme est alors à redéfinir. Censé jouer un rôle prépondérant dans la protection de l'environnement et le développement socio-économique des populations locales, il devrait être en mesure de montrer tous les aspects qui englobent les réalités amazoniennes. Si le toxi-tourisme est déjà largement développé dans différentes régions du monde, notamment en Afrique, il reste limité en Amazonie équatorienne, l'image véhiculée sur cette région depuis maintenant plus de quatre siècles étant intimement liée au style de tourisme proposé aujourd'hui.

L'inquiétude ressentie par les habitants de San Virgilio révèle la vision qu'ils ont eux-mêmes du tourisme et de l'image qui « doit » être véhiculée : satisfaire le client, lui offrir ce pour quoi il est venu... Or, la communauté est directement victime de la présence pétrolière sur ses terres et les futurs touristes seront alors bel et bien confrontés au passage incessant des hélicoptères. San Virgilio se trouve dans une situation très complexe. Dénoncer auprès des touristes les impacts causés par l'entreprise Agip alors que cette dernière est l'investisseur principal de son projet touristique apparaît trés délicat. Les problèmes socio-économiques des communautés confrontées aux pétroliers sont néanmoins une réalité tragique que la majorité des touristes ne voit pas, à la fois parce qu'on leur en donne rarement l'occasion, la plupart étant peu en contact direct avec la population, mais également parce que les activités proposées, orientées sur les aspects naturels et culturels sont très ciblées, orientées, afin que le touriste se remplisse les yeux de « beau » et continue inconsciemment de perpétuer cette image idéalisée de la vie en forêt où tout n'est qu'abondance, calme et plaisirs des sens.

19 L'idée du toxic-tours date de la fin des années 1990 en Amazonie équatorienne, à l'initiative de I'ONG Acción ecológica qui a souhaité montrer les dégâts causés par Texaco dans la province de Sucumbios. Depuis, plusieurs ONG organisent des séjours de 1 à 10 jours proposant des « reality tours » ou « Bici Toxi Tour »... « Ingeniero sin fronteras »(ONG) propose également des toxic-tours d'une journée, gratuits, dans le parc du Yasuní. Le procès de Texaco relancé en 2003 auprès de la cour équatorienne, ainsi que le projet ITT, au centre de l'actualité depuis le sommet de Copenhague en décembre 2009, ne sont certainement pas étrangers à cette nouvelle forme de tourisme qui tend éveille la conscience aux problèmes de contamination environnementale. Le 9 mai 2010, un article sur les toxic-tours est paru dans El Telégrafo, un des quotidiens les plus lus du pays, montrant l'intérêt croissant dont fait preuve cette forme de militantisme touristique amazonien. 


\section{CONCLUSION}

En s'intégrant au marché touristique international, les communautés cherchent à créer des emplois, à développer de nouveaux projets locaux et à encourager la jeune génération à rester sur place. Le tourisme communautaire apparaît ainsi comme la solution miracle, répondant à une problématique économique et sociale complexe à laquelle est confrontée la majorité des indigènes. La nécessité de se réapproprier une culture, de réinventer un patrimoine et de valoriser des traditions perdues fait également partie de ce processus. Ce dernier a tendance à favoriser une nouvelle dynamique locale, à renforcer les liens sociaux et à participer à une redéfinition des valeurs culturelles. II modifie également profondément la manière de concevoir sa propre culture, obligeant à inventer et à négocier sans cesse les formes culturelles que les habitants souhaitent valoriser et montrer. Conscientes du processus de «folklorisation » lié à la pratique du tourisme, les communautés revendiquent néanmoins « l'authenticité » de leur culture qu'elles intègrent à un processus plus large de reconnaissance identitaire sur le plan national et international.

Au-delà des enjeux économiques et identitaires locaux, indéniablement liés à cette activité, cette nouvelle forme de tourisme s'inscrit également dans la droite ligne des politiques internationales de développement (OIT, OMT, Unesco). Parmi tous les projets de développement de la région amazonienne au cours des cinq derniers siècles (exploitation des ressources, voies d'accès, agriculture, éducation...), le tourisme communautaire diffère dans son approche puisqu'il veut donner l'impression aux habitants qu'ils en sont à la fois les initiateurs, les propriétaires et les acteurs. L'indépendance des communautés reste cependant très relative car liée à plusieurs facteurs résultants de la capacité de financement et de la fréquentation touristique. L'échec d'Ishkay-Yaku est de ce point de vue éloquent. L'absence de touristes a suscité un désintérêt certains des habitants pour le projet et provoqué une scission aux seins des dirigeants. Peu de communautés vivent réellement du tourisme et les désillusions peuvent avoir des conséquences sociales néfastes. La construction du complexe de San Virgilio démontre néanmoins le rôle clé joué par le tourisme dans les nouveaux projets de développement locaux et l'espoir d'une vie meilleure qu'il sous-tend.

Dans le cadre de la mise en place de projet de tourisme communautaire, la présence d'acteurs extérieurs est cependant inévitable et, semblerait-il, vitale pour fonctionner sur le marché touristique international. La multiplication de ces acteurs au cours des deux dernières décennies (État, ONG, Agence de voyage, Tours opérateurs, pétroliers...) révèle les enjeux d'une telle activité (Bretón, 2001). Après l'or, le caoutchouc et le pétrole, le tourisme apparaît comme la nouvelle ressource de l'Amazonie (Chaumeil, 2009). L'intervention de la compagnie Agip et le poids important de cette dernière dans les prises de décisions locales jouent un rôle central dans le développement touristique des communautés d'Atacapi, de Liquino et de San Virgilio. Sa participation au projet a cependant créée un phénomène de dépendance, aliénant toute forme d'initiative et modifiant 
profondément les rapports sociaux. Ces communautés ne sont pas un cas isolé et reflètent un mal être perceptible chez la majorité des peuples autochtones. Ici comme partout ailleurs, on assimile la dépendance à l'ennui, laissant le champ libre aux entreprises, se positionnant comme les nouveaux mécènes, « attentifs » au bien-être des populations autochtones. Tout le paradoxe repose sur ce point, et démontre le poids que représente ces compagnies pétrolières en Amazonie équatorienne, qui jouent de leur pouvoir économique pour combler l'absence d'un État actuellement en pleine restructuration. Si le tourisme communautaire est, selon Gallo Villamil, président de la FEPTCE, représentatif de la lutte menée par les peuples indigènes ces dernières années, il apparaît néanmoins, de plus en plus financé par les compagnies d'extraction20. Ces dernières ont pris conscience très rapidement qu'en proposant de financer des projets touristiques, elles s'inscrivaient dans un mouvement en pleine effervescence qui répond aux attentes des communautés et leur ouvrait une nouvelle porte d'accès sur les territoires à exploiter.

Des modifications profondes pourraient néanmoins voir le jour dans les décennies à venir, l'actuel gouvernement équatorien ayant décidé de jouer la carte de l'écologie. En s'affichant comme le premier pays au monde reconnaissant le droit de la Nature dans sa nouvelle constitution (2008), I'État a décidé de geler 3 exploitations pétrolières dans le parc du Yasuní (projet ITT)21. Validé par les Nations Unis en juillet 2010, ce projet inédit, totalement innovant, s'inscrit dans les nouveaux enjeux internationaux de protection et de conservation environnementales. Il consiste à ne pas exploiter $20 \%$ des ressources pétrolières du pays (environ 850 millions de barils), en échange d'une compensation financière de la communauté internationale (Le Quang, 2010). Or vert ou or noir ? Pour un pays qui vit à 50 \% de ses ressources pétrolières, l'enjeu est de taille.

\section{Références citées}

AVALOS MENDEZ, L., 2005 - Développement et changement culturel chez un peuple indigène de l'Amazonie de l'Équateur, Runa. Undercurrent, vol. 2, nº 1 : 9-16.

BRETÓN, V., 2001 - Cooperación al desarrollo y demandas étnicas en los andes ecuatorianos. Ensayos sobre indigenismo, desarrollo rural y neoindigenismo, 278 p. ; Quito: Flacso.

${ }^{20}$ Certaines communautés s'opposent néanmoins aux pétroliers. La communauté de Sarayaku a, par exemple, basé son projet touristique autour de son combat contre l'entreprise Agip (bloc 10) et la compagnie CGC (bloc 23). Ses nombreux contacts avec des institutions ou ONG étrangères et sa présence importante sur la scène internationale lui permettent aujourd'hui de se positionner comme LA communauté qui s'oppose aux compagnies pétrolières en Amazonie équatorienne. Les multiples financements qu'elle obtient à travers le monde participent à son combat tout en lui procurant une indépendance totale.

21 ITT correspond au nom des 3 puits de pétrole exploitables dans la région du parc du Yasuní, zone protégée située dans la province d'Orellana au nord-est du pays : Ishpingo, Tambococha et Tiputini. 
Tourisme communautaire, conflits internes et développement local

CHAUMEIL, J.-P., 2009 - El comercio de la cultura: el caso de los pueblos amazónicos. Bulletin de l'Institut Français d'Études Andines, Tome 38, $\mathbf{n}^{\circ} \mathbf{1}:$ 61-74.

COCA PÉREZ, A., 2007 - Machacuyacu, turismo y organización comunitaria. In : Turismo comunitario en Ecuador. Desarrollo y sostenibilidad social (E. Ruiz Ballesteros \& D. Solis Carrión, eds.) : 93-124; Quito : Abya-Yala.

COLVIN, J., 1994 - Capirona: A model of indigenous ecotourism. Paper delivered at the Second Global Conference: Building a Sustainable World through Tourism ; Montréal, Canada.

DEL CAMPO TEJEDOR, A., 2009 - La autenticidad en el turismo comunitario. Tradición, exotismo, pureza, verdad. In : Cultura, comunidad y turismo. Ensayos sobre el turismo comunitario en Ecuador (E. Ruiz Ballesteros \& M. A. Vintimilla, eds.) : 41116 ; Quito : Abya-Yala.

DRUMM A., 1991 - Integrated Impact Assessment of Nature Tourism in Ecuador's Amazon Region, 62 p. ; Quito : Feprotur.

DRUMM, A., 1998 - New Approaches to Community-based Ecotourism Management, Learning from Ecuador. In : Ecotourism, A Guide for Planners \& Managers, Volume 2 : 197-213; Burlington, VT USA : The International Ecotourism Society.

DUMOULIN, D., 2006 - Les «terres indiennes pour la conservation», un dispositif clé du néo-indigénisme international. In : Être Indien dans les Amériques (C. Gros \& M.-C. Striegler, eds.) : 91-103; Paris : Éditions de l'Institut des Amériques.

EPLER WOOD, M., 1998 - Meeting the global challenge of community participation in ecotourism: case studies and lessons from Ecuador, 32 p. ; Arlington, Virginia : The Nature Conservancy.

EPLER WOOD, M., 2002 - Ecotourism: Principles, Practices and Policies for Sustainability, 64 p. ; New York : United Nations Publications.

FONTAINE, G., 2004 - Actores y lógicas racionales en los conflictos socio-ambientales: el caso del bloque 10 en Ecuador (Pastaza). In : Globalización y desarrollo en América Latina (F. Falconí, M. Hercowitz \& R. Muradián, eds.) :155-172 ; Quito : FLACSO.

FONTAINE, G., 2006 - Petróleo y desarrollo sostenible en el Ecuador. 3. Las ganancias y pérdidas ; Quito : Flacso, ILDIS-FES, Petrobras Ecuador.

GORMAN, M., 2006 - El ecoturismo, una alternativa para el desarrollo sustentable del oriente ecuatoriano, http://www.infoandina.org/sites/default/files/recursos/Oriente Ecuatoriano_Planeta_com.mht

HUTCHINS, F., 2007 - Footprints in the forest: ecotourism and altered meanings in Ecuador's upper Amazon. Journal of Latin American and Caribbean Anthropology, vol. 12, $\mathbf{n}^{\circ} \mathbf{1}:$ 75-103.

KIMERLING, J., 1993 - Crudo amazonico, 152 p. ; Quito : Abya-Yala.

LE QUANG, M., 2010 - Une innovation contre le réchauffement climatique : I'Initiative Yasuní-ITT en Équateur. Mouvements, http://www.mouvements.info/Uneinnovation-dans-la-lutte.html

LENCLUD, G., 1987 - La tradition n'est plus ce qu'elle était... Sur les notions de tradition et de société traditionnelle en ethnologie. Terrain, $\mathbf{n}^{\circ} \mathbf{9}$.

http://terrain.revues.org/index3195.html.

MURATORIO, B., 1991 - The Life and Times of Grandfather Alonso: Culture and History in the Upper Amazon, 312 pp. ; New Brunswick : Rutgers University Press.

MURATORIO, B., 1994 - Imágenes e imagineros. Representaciones de los indígenas ecuatorianos, siglos XIX y XX, 293 pp.; Quito : Flacso- Sede Ecuador. 
MURATORIO, B., 1996 - Trabajando para la Shell: resistencia cultural a la proletarización en la Amazonía ecuatoriana. In: Globalización y cambio en la Amazonía indígena (Fernando Santos Granero, ed.), volumen 1 : 371-396; Quito : FLACSO, Abya-Yala.

NARVAEZ, I., 1996 - Huaorani vs Maxus. Poder étnico vs poder transnacional, 116 p. ; Quito : FESO.

RAMÍREZ GALLEGOS, F., 2009a - La felicidad como medida del buen vivir en Ecuador, 28 p. ; Quito : Secretaria Nacional de Planificación y Desarrollo (Senplades).

RAMíREZ GALLEGOS, F., 2009b - El movimiento indígena y la reconstrucción de la izquierda en Ecuador. In : Los Andes en Movimiento. Identidad y poder en el nuevo paisaje político (P. Ospina, O. Kaltmeier \& C. Büschges, eds.) : 65-94; Quito : Universidad Andina Simón Bolívar, Corporación Editora Nacional, Universidad de Bielefeld.

RIVAL, L., 1997 - Oil and sustainable development in the Latin American humid tropics. Anthropology Today, vol. 13, $\mathbf{n}^{\circ} 6: 1-3$.

RODRÍGUEZ, A., 1999 - Kapawi: a model of sustainable development in Ecuadorian Amazonia. Cultural Survival Quaterly, $\mathbf{n}^{\circ} 23$ : 43-44.

RUIZ BALLESTEROS, E. \& HERNÁNDEZ, M., 2010 - Tourism that Empowers? Commodification and Appropriation in Ecuador's Turismo Comunitario. Critique of anthropology, vol. 30 (2) : 201-229.

RUIZ BALLESTEROS, E. \& SOLIS CARRION, D., 2007 - Turismo comunitario en Ecuador. Desarrollo y sostenibilidad social, 333 p. : Quito : Abya-Yala.

RUIZ BALLESTEROS, E., HERNÁNDEZ, M., COCA, A. \& CAMPO del, A., 2008 - Turismo comunitario en Ecuador. Comprendiendo el community-based tourism desde la comunidad. PASOS, 6 (3) : 399-418.

SANTOS-GRANERO, F., 1992 - Opresión colonial y resistencia indígena en la Alta Amazonía, 184 pp. ; Quito : Flacso, Sede Ecuador.

SANTOS-GRANERO, F., 1994 - Etnohistoria de la Alta Amazonia, siglos XV-XVIIle, 305 p. ; Quito, Abya- Yala. Col. 500 años, n 46.

SANTOS- GRANERO, F., 1996 - Globalización y cambio en la Amazonía indígena, 472 p. ; Quito : Abya-Yala.

SAWYER, S., 1997 - The 1992 indian mobilization in lowland Ecuador. Latin American Perspectives, vol. 24, $\mathbf{n}^{\circ} \mathbf{3}:$ 65-82.

SCHALLER, D., 1996 - Indigenous Ecotourism and Sustainable Development:The Case of Río Blanco, Ecuador, http://www.eduweb.com/schaller/index.html

SMITH, R., 1993 - Drama bajo el manto amazónico: el turismo y otros problemas de los Huaorani en la actualidad, 375 p. ; Quito : Abya-Yala.

STRONZA, A., 2001 - Forging new ground for ecotourism and other alternatives. Annual review of anthropology, vol. $30: 261-283$.

STRONZA A. \& GORDILLO, J., 2008 - Community views of ecotourism. Annals of Tourism research, vol. 35, issue $2:$ 448-468.

TRUJILLO MONTALVO, P., 2001 - Salvajes, civilizados y civilizadores. La amazonia ecuatoriana: el espacio de las ilusiones, 159 p.; Quito : Abya-Yala.

UNESCO, 1982 - Déclaration de Mexico sur les politiques culturelles. Conférence mondiale sur les politiques culturelles, Mexico City, 26 juillet - 6 août.

VARGA, P., 2007 - Ecoturismo en el Ecuador. Un estudio de antropología de turismo, el caso de los Siona, 267 p.; Quito : Abya-Yala. 
Tourisme communautaire, conflits internes et développement local

WESCHE, R., 1993 - Ecotourism and indigenous peoples in the resource frontier of the Ecuadorian Amazon. Yearbook, Conference of Latin American Geographers, 19 : 35-45.

WESCHE, R., 1996 - Developed country environmentalism and indigenous community controlled ecotourism in the Ecuadorian Amazon. Geographische Zeitzchrift, 3/4 : 157-159.

WESCHE, R. \& DRUMM, A., 1999 - Defending our rainforest: a guide to community based ecotourism in the Ecuadorian Amazon, 215 p. ; Quito : Acción Ecológica.

WUNDER, S. 1996 - Ecoturismo, ingresos locales y conservación. El caso de Cuyabeno, 97 p. ; Quito : UICN.

ZEPPEL, H., 2007 - Indigenous Ecotourism: Conservation and Resource Rights. In: Critical Issues in Ecotourism: Confronting the Challenges (J. Higham, ed.) : 308-348 ; Oxford : Elsevier. 